\title{
Evaluation of the osseointegration of dental implants coated with calcium carbonate: an animal study
}

\author{
Yi Liu ${ }^{1, \star}$, Yi Zhou ${ }^{2, \star}$, Tao Jiang ${ }^{2}$, You-De Liang ${ }^{2}$, Zhen Zhang ${ }^{2}$ and Yi-Ning Wang ${ }^{2}$
}

In an attempt to overcome the limitations of titanium in dental and orthopaedic clinical applications, a new method has been developed to prepare calcium carbonate coatings on sandblasted and acid-etched (SA) titanium implants. The purpose of this study was to investigate the effect of calcium carbonate-SA (CC-SA) implants on osseointegration in vivo. The surfaces of SA and CC-SA implants were characterised for surface morphology and surface chemistry. Subsequently, these two kinds of implants were implanted in the femoral condyles of rabbits. The implants were retrieved and prepared for histological and histomorphometric evaluation 1, 2, 4, 8 and 12 weeks after implantation. Significantly higher values of bone-to-implant contact of the entire implant except the gap area (BIC_ALL) and the bone-to-implant contact of the gap area (BIC_GAP) were found in animals with the CC-SA implants than in those with the SA implants at 4 weeks. Higher values of total gap bone were found in those with the CC-SA implants than in those with the SA implants at 1, 2 and 4 weeks. In conclusion, the current findings demonstrate that the calcium carbonate coating can improve and accelerate the early ingrowth of bone and osseointegration at the early healing phase. This may reduce clinical healing times and thus improve implant success rates.

International Journal of Oral Science (2017) 9, 133-138; doi:10.1038/ijos.2017.13; published online 28 April 2017

Keywords: calcium carbonate; histomorphometry; in vivo; osseointegration; titanium

\section{INTRODUCTION}

Titanium is one of the most commonly used materials for dental and orthopaedic implants on account of its high biocompatibility, chemical stability and excellent mechanical properties. ${ }^{1-2}$ However, the oxide film that spontaneously forms on titanium when it is exposed to oxygen makes it bioinert. ${ }^{3}$ Modifications of metal surfaces are often employed as a means of controlling the implant-tissue interaction and osseointergration. ${ }^{4}$

Applying bioactive coatings on titanium implants is one of the most important ways to improve surface properties, ${ }^{5}$ so that the mechanical strength of the titanium implants and the bioactivity of the coatings are well combined. HA-coated surfaces achieve a very intimate bone-to-implant contact and have been claimed to reduce the healing period. However, a retrospective long-term clinical study on 313 HA-coated oral implants reported that the cumulative survival rate decreased to $77.8 \%$ after 8 years for HA-coated implants, compared with $92.7 \%$ for titanium plasma-sprayed implants. ${ }^{6}$ Another clinical report also showed that the cumulative success rates of HA-coated implants after 5 and 10 years were $89.9 \%$ and $54 \%$, respectively. ${ }^{7}$ The long-term effects of HA-coated implants may be limited by vulnerable interface attachment between the HA coating and the implant metal body, ${ }^{8}$ surface resorption-induced foreign body reactions ${ }^{9}$ and relatively low shear and fatigue strengths. ${ }^{10}$

Therefore, the idea of a resorbable coatings was developed based on the thought that an optimal coating should completely disappear before completion of the bone-healing process ${ }^{11}$ and the formation of the bone-titanium contact. Oh et al. ${ }^{12}$ found that thinner coatings have been shown to be as effective as thicker coatings with respect to enhancement of the early bone response. Moreover, degradation of these coatings can even be associated with increased osteoconductivity and enhanced bone-implant contact (BIC). ${ }^{13}$

Both calcium phosphate and calcium carbonate are resorbable in vivo. Biomimetic calcium phosphate is regarded as an ideal coating material; studies have shown that it can promote bone formation. ${ }^{14-15}$ Like calcium phosphate, calcium carbonate has been widely used as a bone substitute for decades. ${ }^{16-17}$ Compared to calcium phosphate, calcium carbonate has a higher solubility. ${ }^{18}$ Therefore, some research has investigated the combination of calcium phosphate and calcium carbonate as a biphasic biomaterial. ${ }^{19}$

Calcium carbonate has been shown to be a biocompatible and osteoconductive material in the form of either aragonite or calcite. ${ }^{20-21}$

\footnotetext{
${ }^{1}$ Department of Stomatology, Huangshi Central Hospital, Affiliated Hopsital of Hubei Polytechnic University, Edong Healthcare Group, Huangshi, China and ${ }^{2}$ The State Key Laboratory Breeding Base of Basic Science of Stomatology (Hubei-MOST) \& Key Laboratory of Oral Biomedicine, Ministry of Education, School and Hospital of Stomatology, Wuhan University, Wuhan, China

*These authors contributed equally to this work.

Correspondence: Professor YN Wang, The State Key Laboratory Breeding Base of Basic Science of Stomatology (Hubei-MOST) \& Key Laboratory of Oral Biomedicine, Ministry of Education, School and Hospital of Stomatology, Wuhan University, 237 Luoyu Road, Wuhan 430079, China

E-mail: wang.yn@whu.edu.cn

Accepted 20 December 2016
} 
Most importantly, calcium carbonate is degradable in vivo, making it a favourable candidate for implant coating, but no report on the use of this material has been available because of the technological difficulty of applying it as a coating. Recently, however, a method was developed to deposit calcium carbonate coatings on silicon wafers. ${ }^{22}$ Inspired by this, we used the new method to apply a calcium carbonate coating to sandblasted and acid-etched titanium (SATi) implants, a clinically successful implant. Our previous research has shown that calcium carbonate coating may yield a better biological outcome than titanium implants in vitro because it may induce differentiation towards an osteoblastic phenotype and therefore enhance the osteointegration process, especially in the early stage. ${ }^{23}$ The aim of the present study was to investigate the characteristics and in vivo bone formation of calcium carbonate-coated implants.

\section{MATERIALS AND METHODS}

\section{Implant preparation}

In total, 36 commercial pure titanium implants were machined for this in vivo study. The dumbbell-shaped implant was $3.3 \mathrm{~mm}$ in diameter and $8 \mathrm{~mm}$ in length. A gap was made in the middle of the implant, $2 \mathrm{~mm}$ long and $0.2 \mathrm{~mm}$ wide. The gap enabled investigation of the new bone formation, which is very difficult to assess on an implant surface that is in contact with the bone of a drilled hole. All implants were sandblasted with $0.25-0.50 \mathrm{~mm}$ corundum grit at 5 bars for $1 \mathrm{~min}$. Subsequently, the implants were acid-etched in hydrochloric acid/sulfuric acid $(1: 1)$ at $65^{\circ} \mathrm{C}$ for $30 \mathrm{~min}$. After the above treatments, the implants were ultrasonically cleaned for $15 \mathrm{~min}$ in acetone, ethanol (70\%) and deionised water, and finally dried at room temperature. Eighteen implants were coated with calcium carbonate; the other 18 implants served as the control.

\section{Calcium carbonate coating}

The experimental setup for preparing the $\mathrm{CaCO}_{3}$ films is illustrated in Figure 1. Two vials-one containing a $20 \mathrm{mmol} \cdot \mathrm{L}^{-1}$ calcium chloride $\left(\mathrm{CaCl}_{2}\right)$ solution, the other containing ammonium carbonate powder -were placed in a desiccator. SATi implants were placed vertically in the $\mathrm{CaCl}_{2}$ solution. $\mathrm{CaCO}_{3}$ films were then deposited on the SATi substrates via slow diffusion of $\mathrm{CO}_{2}$ produced by decomposition of ammonium carbonate at room temperature for $4 \mathrm{~h}$. The samples were rinsed with deionised water for 1 min after deposition and then airdried overnight. Through this process, calcium carbonate-SATi (CC-

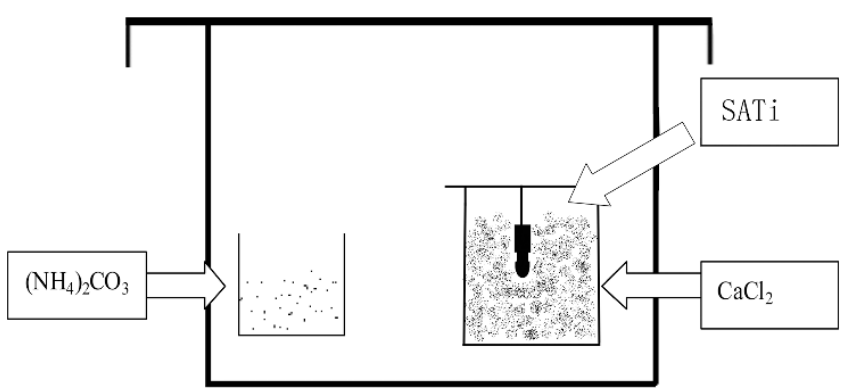

Figure 1 Illustration of the experimental setup for preparation of the calcium carbonate coating on the SA implant surface. Two vials-one containing a $20 \mathrm{mmol} \cdot \mathrm{L}^{-1} \mathrm{CaCl}_{2}$ solution, the other containing ammonium carbonate powder-were placed in a desiccator. SATi implants were placed vertically in the $\mathrm{CaCl}_{2}$ solution. $\mathrm{CaCl}_{2}$, calcium chloride; $\mathrm{SATi}$, sandblasted and acid-etched titanium.
SATi) implants were produced. All implants were sterilised by autoclaving before surgery (for $15 \mathrm{~min}$ at $121^{\circ} \mathrm{C}$ ).

\section{Surface characteristics of implants}

Two test and two control implants were used for surficial analysis.

A field-emission scanning electron microscope (FESEM, JEOL JSM-6700F; JEOL, Tokyo, Japan) was used to investigate the morphology and microstructure of the surfaces at an operating voltage of $5 \mathrm{kV}$. During the Fourier transform infrared (FTIR) test, the coating was scraped from CC-SATi surfaces and collected. The FTIR spectra for the coating were measured by FTIR spectroscopy (Nicolet 170SX; Madison, WI, USA). FTIR bands were identified via comparison with the literature values.

\section{Surgical procedure}

The study protocol was approved by the Ethics Committee for Animal Research, Wuhan University, China. A total of 16 adult male New Zealand white rabbits, $8-12$ months of age and weighing between 2.0 and $2.5 \mathrm{~kg}$, were used in the study. The animals were housed in single boxes under the same environmental conditions $\left(22^{\circ} \mathrm{C} \pm\right.$ $1{ }^{\circ} \mathrm{C}, 55 \% \pm 5 \%$ relative humidity), and they were fed a standard diet and filtered tap water. For surgery, 16 rabbits were anaesthetised with intramuscular injections of SMX compound anaesthesia (Institute of Veterinary, University of PLA, Changchun, China) at a dose of $0.3 \mathrm{~mL} \cdot \mathrm{kg}^{-1}$ body weight. SMX compound anaesthesia consisted of exylazole, ethylene diamine tetraacetic acid, dihydroetorphine hydrochloride and haloperidol. Each rabbit received one SA implant and one CC-SA implant at the same position in the femoral condyles close to the knee joint. All operations were performed by the same experienced implantologist. The antibiotic (ampicillin sodium; $25 \mathrm{mg} \cdot \mathrm{kg}^{-1}$ intramuscularly daily) was administered for 5 days.

One rabbit was complicated by infection and was not included in any of the experimental or control groups. The remaining 15 rabbits survived the treatment and were available for evaluation.

At each time point (1, 2, 4, 8 and 12 weeks post surgery), three animals were killed via intravenous injections of an overdose of SMX compound anaesthesia. The clinical examination performed after death showed that all the implants were clinically stable.

\section{Histological and histomorphometric procedures}

Femoral condyles with implants, processed for ground sectioning, were immediately fixed with $4 \%$ neutral-buffered paraformaldehyde, dehydrated in an ascending series of ethanol concentration, placed in xylene and subsequently embedded in methylmethacrylate in vacuum. Non-decalcified sections were prepared using a slow-speed saw with coolant (Leica SP1600; Leica Spa, Milan, Italy). These sections were made in a transversal direction perpendicular to the axis of the implant. Sections were then ground to a final thickness of $20 \mu \mathrm{m}$ and stained with basic fuchsin and methylene blue.

Three sections were taken from each specimen for histological and histomorphometrical evaluation. An independent examiner performed histological analysis using a microscope equipped with an imaging system (Q-500 MCA; Leica, Wetzlar, Germany). Photographs of histomorphometric analysis were acquired using a computer-based National Institutes of Health image analysis system.

The histomorphometrical analysis consisted of the following measurements. 
1. The amount of bone contact at the interface (BIC): the percentage of implant length showing a direct bone-to-implant contact without any intervening soft tissue layer. The parameter was further differentiated into the percentage of bone-to-implant contact of the entire implant except the gap area (BIC_ALL) and the percentage of bone-to-implant contact of the gap area (BIC_GAP). The gap area, which was in the middle of the implant, was $2 \mathrm{~mm}$ long and $0.2 \mathrm{~mm}$ wide.

2. Total gap bone (TGB): the amount of bone inside the gap between the implant and the cancellous bone.

3. Bone volume/tissue volume (BT05): percent bone area at a distance of $0.5 \mathrm{~mm}$ from the implant surface except the gap area.

\section{Statistical analysis}

Statistical analysis was performed using SPSS 10.0 for Windows (Seattle, WA, USA). Data are reported as the mean \pm standard deviation at a significance level of $P<0.05$. Paired $t$-tests were used for the comparison of parameters between the SA and CC-SA groups at the same healing time.

\section{RESULTS}

Surface characterisation

FESEM micrographs of the SATi and CC-SATi surfaces are shown in Figure 2. SATi surfaces contained pits and craters (Figure 2a). The pits were $0.5-3 \mu \mathrm{m}$ in diameter and appeared to coalesce to form large craters. Crystals with a rhombohedral and needle-like shape were observed on the CC-SATi surfaces (Figure 2b). They formed discontinuous coatings on the substrate. Some crystals partly contacted each other, and some were separated from the neighbouring crystals. Gaps between the separated crystals ranged from several $\mu \mathrm{m}$ to $20 \mu \mathrm{m}$.

The FTIR spectra of crystals deposited on CC-SATi are presented in Figure 3. The precipitates were mainly a mixture of calcite and aragonite. Two sharp absorption bands were found at 876 and $713 \mathrm{~cm}^{-1}$, which was the characteristic frequency of well-formed calcite. The spectra also displayed the characteristic absorption peaks of aragonite at 855,713 and $700 \mathrm{~cm}^{-1}$.

\section{Experimental animals}

During the experiment, all 15 rabbits remained in good health and did not show any wound complications. A total of 30 implants were retrieved after the animals were killed. The implant sites showed no sign of inflammation, and the implants were clinically stable at the time of harvest.

\section{Histology results}

Light micrographs of the bone-implant interface are shown in Figures 4 and 5. At 1 week, there was no obvious difference between the CC-SA and SA implant at high magnification. A gap between the old bone and the implant surface was observed in most areas. At 2 weeks, new bone formation was present around all implants in the cancellous bone. In both groups, many cubical osteoblasts were present and contacted the bone matrix near the implant surface. At 4 weeks, bone modelling was observed for the two kinds of implants, where lamellar bone was observed surrounding an abundant vascular structure. There seemed to be more osteoblasts in the area near the

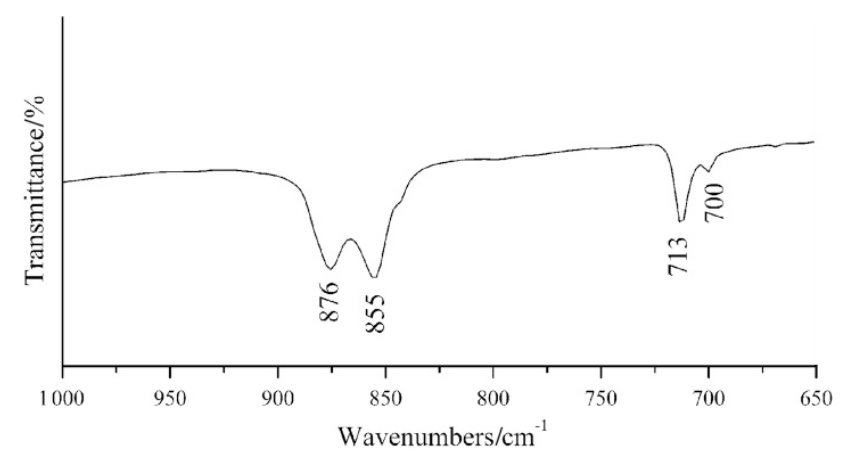

Figure 3 Fourier transform infrared spectra of $\mathrm{CaCO}_{3}$ crystals deposited on the sandblasted and acid-etched titanium surface.
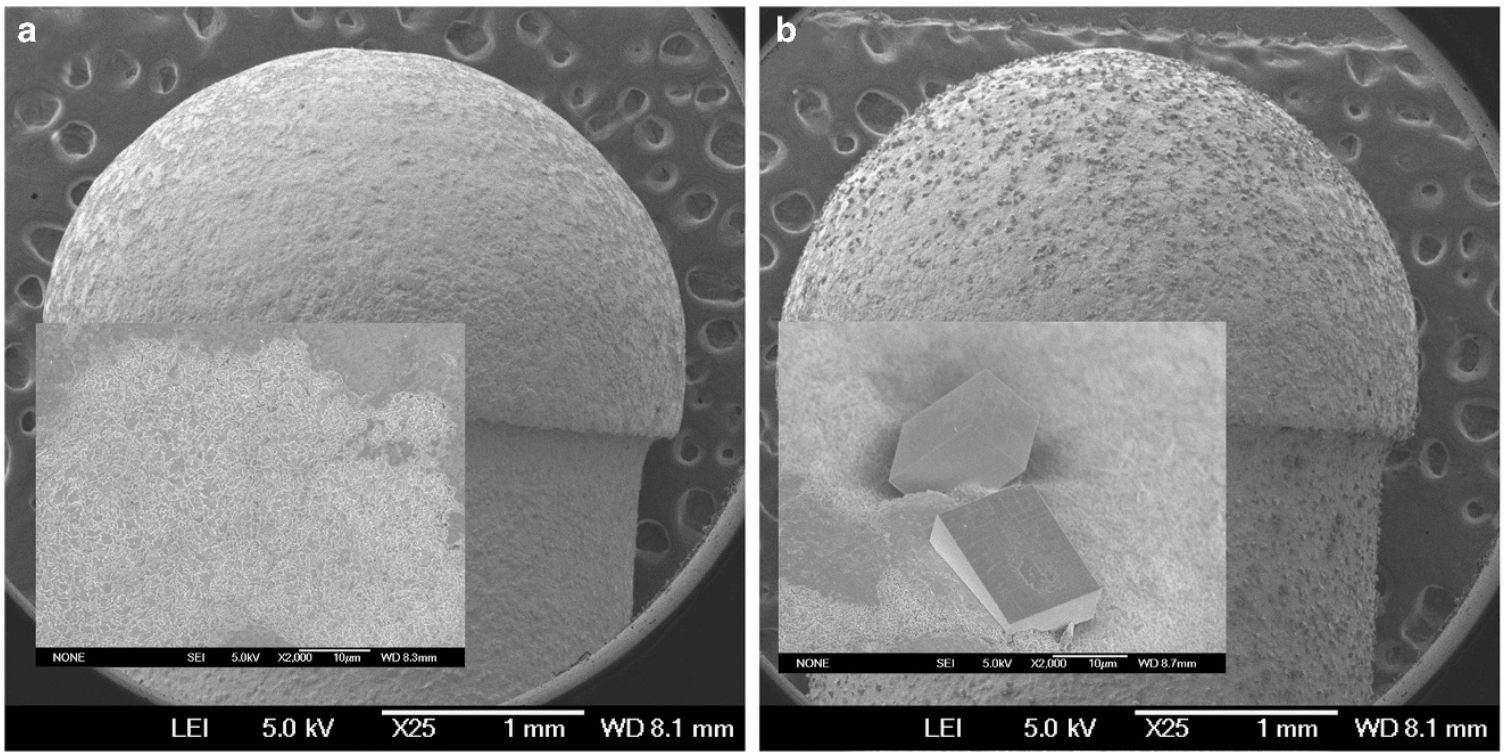

Figure 2 Field-emission scanning electron microscope micrographs showing the surface topography of the tested titanium implants. (a) SATi; (b) CC-SATi. CC-SATi, calcium carbonate-SATi; SATi, sandblasted and acid-etched titanium. 

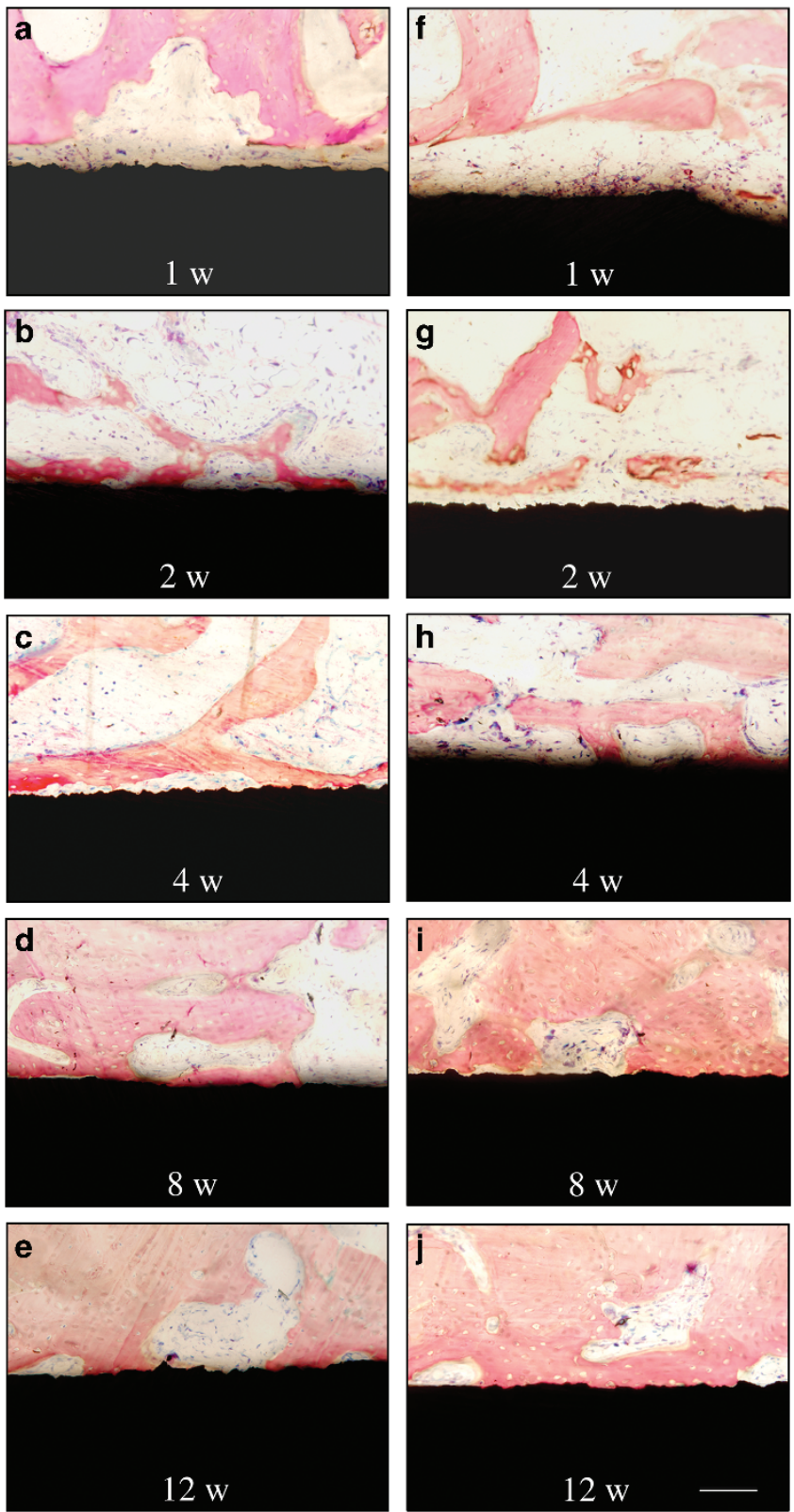

Figure 4 Light micrographs of bone-implant interfaces. SA implants implanted in rabbits at 1 (a), 2 (b), 4 (c), 8 (d), 12 weeks (e) and CC-SA implants implanted in rabbits at 1 (f), 2 (g), 4 (h), 8 (i), 12 weeks (j). Basic fuchsin and methylene blue staining, $\times 20$. Scale bar $=100 \mu \mathrm{m}$. CC-SA, calcium carbonate-SA; SA, sandblasted and acid-etched.

surface of the CC-SA implant than near the SA implant. At 8 weeks, Haversian systems were observed for both groups. At 12 weeks, mature bone with well-mineralised osteoid was present in both groups. Flat lining cells were observed on the surface of trabeculae.

\section{Histomorphometric results}

All bone apposition data for the two kinds of materials are shown in Figure 6. Significantly higher values of BIC_ALL were found in the SA implants than in the CC-SA implants at 1 week, but at 4 weeks they were higher in the CC-SA implants than in the SA implants (Figure 6a). At 1 and 2 weeks, significantly higher values of BIC_GAP
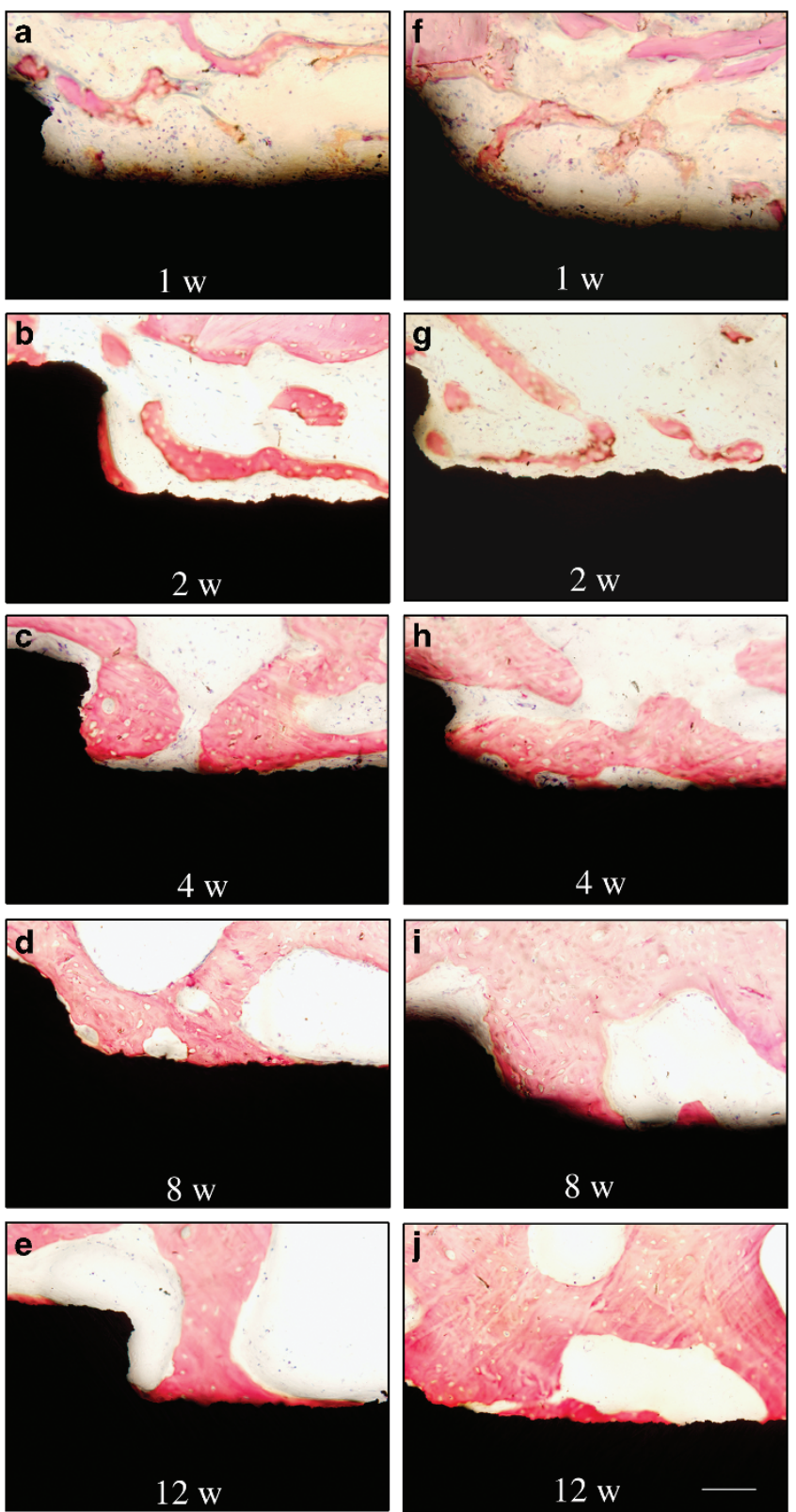

Figure 5 Light micrographs of the gaps. SA implants implanted in rabbits at 1 (a), 2 (b), 4 (c), 8 (d), 12 weeks (e) and CC-SA implants implanted in rabbits at 1 (f), 2 (g), 4 (h), 8 (i), 12 weeks (j). Basic fuchsin and methylene blue staining, $\times 20$. Scale bar $=100 \mu \mathrm{m}$. CC-SA, calcium carbonate-SA; SA, sandblasted and acid-etched.

were found in the SA implants than in the CC-SA implants (Figure 6b). In comparison with the values for the SA implants, higher values of BIC_GAP were found in the CC-SA group at 4 weeks. The BIC_ALL and BIC_GAP values showed no statistically significant difference at 8 and 12 weeks.

The data for the bone volume/tissue volume near the implant surface (BT05) are shown in Figure 7. There were no significant differences between CC-SA and SA surfaces at any of the time points. A higher value of TGB, an index of new bone formation, was found in the CC-SA implants than in the SA implants at 1,2 and 4 weeks but not at 8 and 12 weeks. (Figure 8 ). 
a

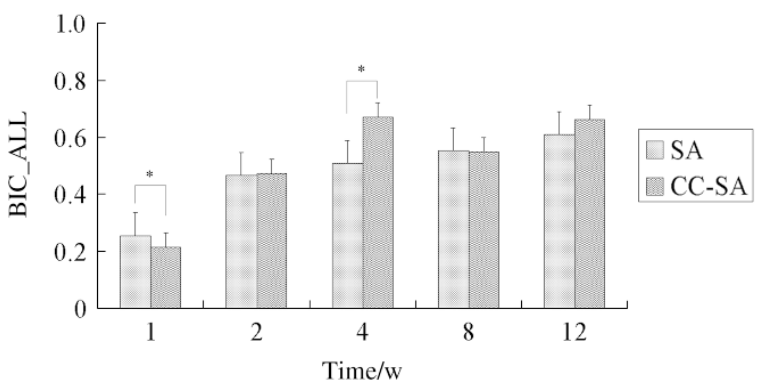

b



Figure 6 Bone-to-implant contact, expressed as the percentage of implant length showing a direct bone-to-implant contact without any intervening soft tissue layer. The parameter was further differentiated into (a) the percentage of bone-to-implant contact of the entire implant except the gap area (BIC_ALL) and (b) the percentage of bone-to-implant contact of the gap area (BIC_GAP). Error bars represent means \pm standard deviation for $n=6$. ${ }^{*} P<0.05$, ${ }^{*} P<0.01$. CC-SA, calcium carbonate-SA; BIC, bone-to-implant contact; SA, sandblasted and acid-etched.

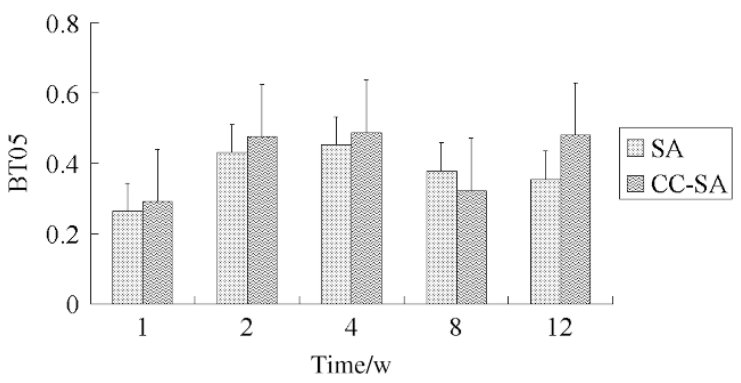

Figure 7 Bar graph showing the percent bone area at a distance of $0.5 \mathrm{~mm}$ from the implant surface except the gap area (BT05). Significant differences were not found between CC-SA and SA implants. Error bars represent means \pm standard deviation for $n=6$. CC-SA, calcium carbonate-SA; SA, sandblasted and acid-etched.

\section{DISCUSSION}

Our idea for developing an implant coating was to construct a resorbable layer that accelerates new bone formation at the early phase of osseointegration and disappears before the bone makes contact with the titanium. This idea is based on the clinical observation that the stability of implants is significantly decreased at 3-4 weeks post surgery compared to readings obtained at surgery, but subsequently increases. $^{24-25}$ Secure primary stabilisation followed by robust osseointegration are of critical importance for the clinical result and the longterm stability of the implant. ${ }^{26}$ The aim of the calcium carbonate coating is to minimise the period of reduced stability and promote osseointegration at the early healing stage.

We found that calcium carbonate-coated surfaces significantly enhanced bone-to-implant contact (BIC_ALL) at 4 weeks. Furthermore, the new bone formation in the gap (BIC_GAP) was also higher than in the SA implants at 2 and 4 weeks, which is consistent with previous reports. ${ }^{16-17,20-21}$ The results suggest that the enhanced boneto-implant contact may have been due to superior new bone formation in the calcium carbonate group. The positive effect of the calcium carbonate coating may be based on the stimulation of integrin-mediated osteoblast response by calcium ions through the enhanced ligand binding of the integrin receptor. ${ }^{27-28}$ Physical effects such as changes in surface energy due to implant coating and enlargement of the surface area may also play a role. ${ }^{29}$ Surface roughnesses and the contact angles of CC-SATi and SATi surfaces were measured in our in vitro research. We observed that calcium carbonate crystals, especially rhombohedral ones, made the CC-SATi

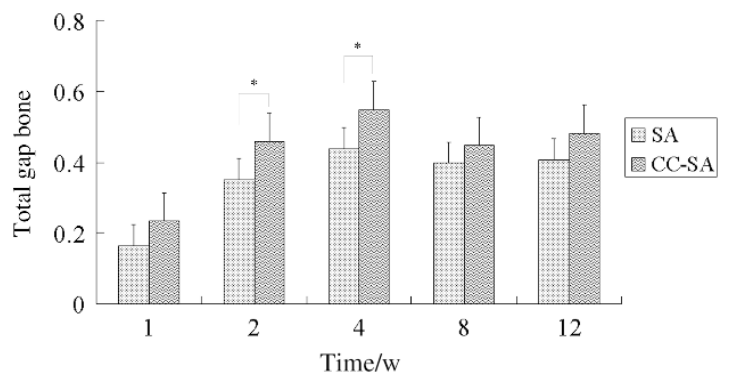

Figure 8 Bar graph showing the amount of bone inside the gap between the implant and the cancellous bone. The TGB in the CC-SA implants was significantly higher than in the SA implants at 1,2 and 4 weeks. Error bars represent means \pm standard deviations for $n=6$. ${ }^{*} P<0.05$, ${ }^{* *} P<0.01$. CC$\mathrm{SA}$, calcium carbonate-SA; SA, sandblasted and acid-etched; TGB, total gap bone.

surfaces rougher than SATi surfaces. However, no significant differences in their contact angles were observed between CC-SATi and SATi. ${ }^{23}$ High surface energy, considered an important variable, was found to be insufficient to cause a marked increase in osteoblast responses to $\mathrm{Ti}$ substrates with low surface roughness. In contrast, when substrates with complex micron-scale and submicron-scale roughness are fabricated to retain the high surface energy of uncontaminated $\mathrm{TiO} 2$, the cells exhibit synergistic enhancement of their response compared to the surface topography alone. ${ }^{30}$

In this study, calcium carbonate coatings were deposited on SA implants using a simple chemical treatment. The SA implants performed well owing to their original titanium properties. ${ }^{31}$ The microporous surface of titanium shows a large peak-to-valley pore height and is thought to increase cell attachment to promote osteoblast differentiation. ${ }^{32}$ Once a CC-SA implant improves peri-implant bone regeneration beyond the effect of the SA implant alone, it can be assumed that a CC-SA implant is an optional material for clinical use.

However, the opposite results were found for BIC_ALL at 1 week and BIC_GAP at 1 and 2 weeks. An explanation for this might be that at an early healing time, some bone directly adjacent to the calcium carbonate was not included in the BIC.

It should be noted that the bond strength of the coating could not be appropriately evaluated using conventional methods such as a shear, tensile or scratch test because the coating was discontinuous. In addition, the thickness is hard to measure. During the FTIR test, we found that it was difficult to scrape the coating from the CC-SATi 
surfaces. Moreover, in this experiment, the calcium carbonate-coated implants were found to significantly enhance bone-to-implant contact (BIC_ALL) at 4 weeks. The new bone formation in the gap (BIC_GAP) was also higher than in the SA implants at 2 and 4 weeks. This indicated that the calcium carbonate coating could withstand peeling during the implant procedure. The bond strength is sufficient for clinical use.

Implants observed at 8 and 12 weeks did not show any significant difference in BIC_ALL and BIC_GAP. This may indicate that the coating was resorbed and the enhancing effect of the calcium carbonate-coated implants as compared with the SA implants was limited to early stages of peri-implant bone formation.

In previous studies, Hernández-Hernández et al. ${ }^{33}$ prepared calcium carbonate using a vapour-diffusion technique. ${ }^{33}$ Isfisco et al. ${ }^{34}$ prepared calcium phosphate nanoparticles using the same technique. Further study is needed to determine whether this technique can be used to deposit calcium phosphate on SA implants and to assess the biological performances of these two kinds of implants.

The FESEM images showed that the titanium surface was only partially covered by the calcium carbonate crystals using the present coating technique. Therefore, our method needs to be improved to achieve an evenly coated calcium carbonate layer. Another limitation of this pilot study was the small number of animals.

\section{CONCLUSIONS}

Our results confirm that CC-SA and SA implants show good biocompatibility and osteoconductive properties after a 12-week healing period. A calcium carbonate coating can improve and accelerate the early ingrowth of bone and osseointegration. This may reduce clinical healing times and thus improve implant success rates. Furthermore, the technique used here is a promising new method to deposit $\mathrm{CaCO}_{3}$ coatings onto titanium substrates and warrants further investigation.

\section{ACKNOWLEDGEMENTS}

This work was funded by the National Natural Science Foundation of China (nos 81200812 and 81371170), the Foundation of Health and the family planning commission of Hubei province (no. WJ2015MB276).

1 Nebe JB, Mueller L, Luethen F et al. Osteoblast response to biomimetically altered titanium surfaces. Acta Biomater 2008; 4(6): 1985-1995.

2 Bacchelli B, Giavaresi G, Franchi MA et al. Influence of a zirconia sandblasting treated surface on peri-implant bone healing: an experimental study in sheep. Acta Biomater 2009; 5(6): 2246-2257.

3 Park JW, Suh JY, Chung HJ. Effects of calcium ion incorporation on osteoblast gene expression in MC3T3-E1 cells cultured on microstructured titanium surfaces. J Biomed Mater Res A 2008; 86(1): 117-126.

4 Kokubo T, Kim HM, Kawashita M. Novel bioactive materials with different mechanical properties. Biomaterials 2003; 24(13): 2161-2175.

5 Langhoff JD, Voelter K, Scharnweber D et al. Comparison of chemically and pharmaceutically modified Titanium and zirconia implant surfaces in dentistry: a study in sheep. Int J Oral Maxillofac Surg 2008; 37(12): 1125-1132.

6 Wheeler SL. Eight-year clinical retrospective study of titanium plasma-sprayed and hydroxyapatite-coated cylinder implants. Int J Oral Maxillofac Implants 1996; 11(3): 340-350.

7 Artzi Z, Carmeli G, Kozlovsky A. A distinguishable observation between survival and success rate outcome of hydroxyapatite-coated implants in 5-10 years in function. Clin Oral Implants Res 2006; 17(1): 85-93.

8 Cook SD, Kay JF, Thomas KA et al. Interface mechanics and histology of titanium and hydroxylapatite-coated titanium for dental implant applications. Int J Oral Maxillofac Implants 1987; 2(1): 15-22.
9 Salvi GE, Lang NP. Changing paradigms in implant dentistry. Crit Rev Oral Biol Med 2001; 12(3): 262-272.

10 Lemons JE. Hydroxyapatite coatings. Clin Orthop Relat Res 1988; 235: 220-223.

11 Barrère $\mathrm{F}$, van der Valk CM, Dalmeijer RA et al. In vitro and in vivo degradation of biomimetic octacalcium phosphate and carbonate apatite coatings on titanium implants. J Biomed Mater Res A 2003; 64(2): 378-387.

12 Oh S, Tobin E, Yang YZ et al. In vivo evaluation of hydroxyapatite coatings of different crystallinities. Int J Oral Maxillofac Implants 2005; 20(5): 726-731.

13 Yamamoto $\mathrm{H}$, Shibata $\mathrm{Y}$, Tachikawa $\mathrm{T}$ et al. In vivo performance of two different hydroxyapatite coatings on titanium prepared by discharging in electrolytes. J Biomed Mater Res B Appl Biomater 2006; 78(1): 211-214.

14 Le Guéhennec L, Soueidan A, Layrolle P et al. Surface treatments of titanium dental implants for rapid osseointegration. Dent Mater 2007; 23(7): 844-854.

15 Stadlinger B, Pilling E, Huhle $\mathrm{M}$ et al. Evaluation of osseointegration of dental implants coated with collagen, chondroitin sulphate and BMP-4:an animal study. Int J Oral Maxillofac Surg 2008; 37(1): 54-59.

16 Piattelli A, Podda G, Scarano A. Clinical and histological results in alveolar ridge enlargement using coralline calcium carbonate. Biomaterials 1997; 18(8): 623-627.

17 Yukna RA, Yukna CN. A 5-year follow-up of 16 patients treated with coralline calcium carbonate (Biocoral) bone replacement grafts in infrabony defects. J Clin Periodontol 1998; 25(12): 1036-1040.

18 Brečević L, Nielsen AE. Solubility of amorphous calcium carbonate. J Cryst Growth 1989; 98(3): 504-510.

19 Combes C, Bareille R, Rey C. Calcium carbonate-calcium phosphate mixed cement compositions for bone reconstruction. J Biomed Mater Res A 2006; 79(2): 318-328.

20 Ripamonti $U$, Crooks J, Khoah $\mathrm{L}$ et al. The induction of bone formation by coralderived calcium carbonate/hydroxyapatite constructs. Biomaterials 2009; 30(7): 1428-1439.

21 Fricain JC, Bareille R, Ulysse $\mathrm{F}$ et al. Evaluation of proliferation and protein expression of human bone marrow cells cultured on coral crystallized in the aragonite or calcite form. J Biomed Mater Res 1998; 42(1): 96-102.

22 Xu XR, Han JT, Cho K. Formation of amorphous calcium carbonate thin films and their role in biomineralization. Chem Mater 2004; 16(9): 1740-1746.

$23 \mathrm{Liu} \mathrm{Y}$, Jiang T, Zhou $\mathrm{Y}$ et al. Evaluation of the attachment, proliferation, and differentiation of osteoblast on a calcium carbonate coating on titanium surface. Mater Sci Eng C 2011; 31(5): 1055-1061.

24 Ersanli S, Karabuda C, Beck F et al. Resonance frequency analysis of one-stage dental implant stability during the osseointegration period. J Periodontol 2005; 76(7): 1066-1071.

25 Nedir R, Bischof M, Szmukler-Moncler S et al. Predicting osseointegration by means of implant primary stability. Clin Oral Implants Res 2004; 15(5): 520-528.

26 Xue WC, Liu XY, Zheng XB et al. In vivo evaluation of plasma-sprayed titanium coating after alkali modification. Biomaterials 2005; 26(16): 3029-3037.

27 Nayab SN, Jones FH, Olsen I. Human alveolar bone cell adhesion and growth on ionimplanted titanium. J Biomed Mater Res A 2004; 69(4): 651-657.

28 Ellingsen JE. A study on the mechanism of protein adsorption to TiO2. Biomaterials 1991; 12(6): 593-596.

29 Schliephake H, Aref A, Scharnweber D et al. Effect of modifications of dual acid-etched implant surfaces on periimplant bone formation. Part II: calcium phosphate coatings. Clin Oral Implants Res 2009; 20(1): 38-44.

30 Zhao G, Schwartz Z, Wieland M et al. High surface energy enhances cell response to Titanium substrate microstructure. J Biomed Mater Res A 2005; 74(1): 49-58.

31 Zhou Y, Jiang T, Qian MB et al. Roles of bone scintigraphy and resonance frequency analysis in evaluating osseointegration of endosseous implant. Biomaterials 2008; 29(4): 461-474.

32 Sammons RL, Lumbikanonda N, Gross M et al. Comparison of osteoblast spreading on microstructured dental implant surfaces and cell behaviour in an explant model of osseointegration. Clin Oral Implants Res 2005; 16(6): 657-666.

33 Hernández-Hernández A, Rodríguez-Navarro AB, Gómez-Morales J et al. Influence of model globular proteins with different isoelectric points on the precipitation of calcium carbonate. Cryst Growth Des 2008; 8(5): 1495-1502.

34 lafisco M, Morales JG, Hernández-Hernández MA et al. Biomimetic carbonatehydroxyapatite nanocrystals prepared by vapor diffusion. Adv Eng Mater 2010; 12(7): B218-B223.

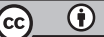

This work is licensed under a Creative Commons Attribution 4.0 International License. The images or other third party material in this article are included in the article's Creative Commons license, unless indicated otherwise in the credit line; if the material is not included under the Creative Commons license, users will need to obtain permission from the license holder to reproduce the material. To view a copy of this license, visit http://creativecommons.org/licenses/by/4.0/

(C) The Author(s) 2017 